\title{
Féeries
}

Études sur le conte merveilleux, XVII $-\mathrm{XIX}{ }^{\mathrm{e}}$ siècle

\section{Les charmes de la physique selon François-Joseph Hunauld : machines-cadavres et merveilleux végétal}

The Charms of Physics according to F.-J. Hunauld: machine corpses and vegetal wonders

\section{Sarah Benharrech}

\section{OpenEdition}

Journals

Édition électronique

URL : http://journals.openedition.org/feeries/1577

DOI : $10.4000 /$ feeries. 1577

ISSN : 1957-7753

Éditeur

UGA Éditions/Université Grenoble Alpes

Édition imprimée

ISBN : 978-2-37747-075-4

ISSN : $1766-2842$

Référence électronique

Sarah Benharrech, "Les charmes de la physique selon François-Joseph Hunauld : machines-cadavres et merveilleux végétal », Féeries [En ligne], 15 | 2018, mis en ligne le 14 février 2019, consulté le 08 septembre 2020. URL : http://journals.openedition.org/feeries/1577 ; DOl : https://doi.org/10.4000/ feeries. 1577

Ce document a été généré automatiquement le 8 septembre 2020

(c) Féeries 


\title{
Les charmes de la physique selon François-Joseph Hunauld : machines-cadavres et merveilleux végétal
}

\author{
The Charms of Physics according to F.-J. Hunauld: machine corpses and vegetal \\ wonders
}

Sarah Benharrech

Il est tentant pour le critique actuel d'adopter le point de vue de Fontenelle lorsqu'il rejette le merveilleux dans les textes qui rendent compte de phénomènes naturels à la fin du XVII et au début du XVIII siècle ${ }^{1}$, pour la raison que ce "faux merveilleux", reconnaissable aux plaisirs de l'imagination qu'il procure et aux rapports ambigus qu'il entretient avec la fable, est source de fausses vérités et de superstition. On lui oppose parfois le "merveilleux vrai» de Réaumur, que révèlent l'examen analytique et la pratique de l'observation directe, et qui engage de la part des observateurs un jugement fondé en méthode et validé par l'expérience. Malheureusement, cette dichotomie, toute séduisante qu'elle puisse paraître, échoue, par son clivage, à représenter la variété des formes de merveilleux en interaction avec les modes de scientificité du XviII ${ }^{e}$ siècle. D'abord, Nathalie Vuillemin note que même le philosophe ne dédaigne pas de susciter l'amour du « faux » merveilleux quand il met en scène une nature selon des "fins rhétoriques, pédagogiques et philosophiques bien précises ${ }^{2}$ ». Ensuite, le merveilleux tient pour beaucoup de la discipline dont il relève, selon que la science est mathématisable, expérimentale ou spéculative. Ainsi, le merveilleux de la physique cartésienne ou celui tiré de l'astronomie diffèrera de celui de la chimie. Il y aurait donc une pluralité de merveilleux, que l'auteur cultive à partir des ressources discursives et analogiques propres à chaque science.

1 L'analogie connaît une fortune contrastée au XVIII ${ }^{\mathrm{e}}$ siècle ${ }^{3}$ bien qu'elle ne cesse d'être utilisée à des fins épistémologiques, didactiques ou argumentatives. L'analogie, selon l'article de Dumarsais dans l'Encyclopédie, désigne «le rapport ou la proportion que 
plusieurs choses ont les uns avec les autres ", et elle est fondée sur une " comparaison ou rapport de ressemblance $»^{4}$. En tant que telle, rapporte Judith Schlanger, elle «fournit des expressions, des arguments, des représentations, des modèles; elle procure à la pensée un support imaginatif ${ }^{5} »$. Outil de représentation, l'analogie rend les concepts perceptibles par les sens ${ }^{6}$. En somme, son usage mobilise les capacités de l'esprit informé par le corps. L'analogie est critiquée par les tenants du rationalisme, qui se méfient des images, même si le mécanisme a proposé ses propres analogies tirées des machines et automates. Les plus empiriques des naturalistes s'en tiennent à un usage fonctionnel et opératoire de l'analogie, dont les termes sont strictement limités dans le cadre d'une hypothèse de travail scientifique précis. Ainsi, Mariotte et Perrault transposent le système circulatoire des animaux aux plantes ; le botaniste Duhamel du Monceau étudie la croissance des branches comparée à celles des os.

2 Le merveilleux tiré de l'analogisme alchimique semble donc avoir été définitivement remisé au rayon des vieilleries littéraires. Pourtant, certains auteurs s'engagent volontairement dans cette voie et remettent à l'honneur une terminologie, une cosmologie et des images considérées comme désuètes. Ces auteurs sont souvent médecins et de ce fait sont préoccupés par le corps vivant. On peut citer pour exemple l'œuvre de Tiphaigne de La Roche, désormais bien connue ${ }^{7}$. Le roman chimique et médical de François-Joseph Hunauld, le Nouveau Traité de physique sur toute la nature publié en 1742, apporte une illustration méconnue de ce réinvestissement apparemment anachronique dans le merveilleux analogique.

3 Par les références à Descartes, Gassendi, Fontenelle et Pluche qui s'entremêlent avec des souvenirs d'Hippocrate, Paracelse, Van Helmont et Campanella, l'ouvrage d'Hunauld semble en effet appartenir à un genre "inactuel». Ce roman à la fois mécaniste et chimique ne fait d'ailleurs aucune mention de la dernière préoccupation des chimistes de son temps, le phlogiston, ce qui pourrait expliquer l'oubli dans lequel ce «roman physico-médicinal» a vite sombré8. Construit sur le modèle du voyage imaginaire et constitué d'entretiens didactiques, le Nouveau Traité de physique appartient au genre hybride des «fictions à vocation scientifique » étudié par Guilhem Armand ${ }^{9}$. On y relève entre autres des traits empruntés aux Entretiens sur la pluralité des mondes de Fontenelle et au Spectacle de la nature de l'abbé Pluche. Peuplé de génies, d'allégories et de divinités de la mythologie, le Nouveau Traité recourt à un merveilleux que l'on pourrait qualifier de "faux». Un premier examen montre toutefois qu'Hunauld fait un usage extrêmement maîtrisé et restreint de ce registre fabuleux alors tombé en désuétude. L'intérêt de son ouvrage se situe ailleurs, nous semble-t-il. Hunauld fonde son enseignement sur une explication mécaniste du monde, dont il sollicite les pouvoirs d'émerveillement, par la mise en scène du dévoilement des rouages secrets, dans la réduction à quelques principes simples et généraux et dans la révélation de l'ordre derrière le chaos des phénomènes. C'est de ce merveilleux mécaniste qu'il tire la matière première de sa fiction. Or Hunauld est médecin et non physicien et, comme nous le verrons, il se heurte rapidement aux limites scénographiques du mécanisme cartésien, plus apte à décrire des automates qu'à mettre en lumière les processus vitaux du corps humain. Son désir de représenter le corps vivant, objet premier de sa pratique médicale, l'appelle alors à explorer l'imaginaire informé par la chimie. Il y trouve, - c'est là que réside pour nous l'intérêt de sa démarche -, des analogies et des correspondances qui évoquent la nature des alchimistes et des astrologues, et dans lesquelles, curieusement, le végétal tient une 
place prépondérante. Curieusement, car les plantes ne cessent alors d'être physiologiquement subsumées au règne animal, tant par la découverte de la reproduction sexuée et de sa reprise dans la classification linnéenne, que par celle du polype. Pourquoi utiliser des images végétales et en quoi correspondent-elles, mieux que la machine, au savoir sensible du médecin? Notre hypothèse est qu'Hunauld oppose la plante à la machine, parce qu'elle est non démontable en ses organes et parce qu'elle résiste à l'abstraction mécaniste. Rendant à l'âme végétative son sens plein d'être vivant, Hunauld ferait par conséquent du végétal l'objet emblématique d'une connaissance sensible du vivant et une source de merveilleux.

\section{De nouveaux entretiens}

Lors de la publication du Nouveau Traité de physique ${ }^{10}$ en 1742 , Hunauld ${ }^{11}$ est au terme d'une brillante carrière en ostéologie, anatomie et médecine. On a de lui des dissertations médicales, des rapports publiés dans les mémoires de l'Académie royale des sciences, et trois opuscules ${ }^{12}$, qui ont ponctué une controverse engagée avec l'anatomiste Jean-Louis Petit ${ }^{13}$ entre 1726 et 1737. Le titre ambitieux de son dernier ouvrage annonce la volonté de donner du monde physique une vision globale, la physique désignant ici l'étude de la nature dans ce qu'elle a de concret, d'observable et d'intelligible. En le qualifiant de "nouveau ", l'auteur reconnaît que son ouvrage vient s'ajouter à une série de traités de physique parmi lesquels on mentionnera en particulier le Traité de physique (1671) de Jacques Rohault ${ }^{14}$, qui entreprend de diffuser les principes de la physique cartésienne. Le médecin Hunauld a choisi le format de l'entretien didactique, entre un maître et son disciple et, comme le révèle le sous-titre - Méditations, et songes sur tous les corps dont la Médecine tire les plus grands avantages pour guérir le corps humain dans lequel l'auteur promet que l'on verra plusieurs curiosités qui n'ont point paru -, la dimension onirique colore l'ensemble d'un fabuleux absent du traité de Rohault. C'est d'ailleurs le songe qui structure la narration. Le récit est motivé par une fiction-cadre où les personnages, la temporalité et l'espace sont conformes aux exigences du vraisemblable. Un jeune homme part à la chasse avec des amis. Se retrouvant seul dans la forêt, il découvre un champ où fleurissent des pavots. Leur effet ne tarde pas à se faire sentir ; il s'endort d'un profond sommeil. À un second niveau du récit, le narrateur endormi fait irruption dans « des Jardins enchantés, aussi beaux que ceux des Fées, et tels que la Fable nous les représente ${ }^{15}$ ». Puis, en compagnie d'un Génie, il entre dans un superbe château où il rencontre les divinités Nature et Histoire. Plus tard, il aura le privilège de converser avec Flore, Démogorgon ${ }^{16}$ et Prométhée, puis de visiter le laboratoire de Chimie. En signe de rite initiatique, car le rêve devient seconde naissance, le jeune homme, anonyme dans la fiction-cadre, reçoit le nom d'Asclépiade. Chaque songe prend fin avec le réveil du jeune homme qui n'a alors d'autre souci que de se replonger dans ses rêves. L'entretien savant commence lors du second songe, et porte sur les mouvements de l'air et les opérations chimiques des gaz, des minéraux et des métaux. Dans le songe suivant, Asclépiade rencontre Flore, que le Génie prie de dévoiler ses secrets sur les plantes et la faculté végétative. Enfin, dans le dernier, Prométhée et Chimie enseignent à Asclépiade la digestion et la circulation du sang, en présence du Génie, d'Anatomie et de Mécanique. L'ouvrage prend fin quand le disciple se réveille.

4 En mettant en place une scénographie comparable ${ }^{17}$ à celle des Entretiens sur la pluralité des mondes, Hunauld continue la veine creusée par Fontenelle, mais il déséquilibre 
davantage le rapport d'autorité entre le maître et le disciple. Dans les Entretiens, un philosophe présente le système solaire à une marquise, dont les questions et les réflexions font honneur à sa vive intelligence. La marquise tient lieu de disciple et son ignorance sert de prétexte à des explications aux images frappantes, le philosophe utilisant de fréquentes métaphores et analogies pour rendre sensibles les ressorts cachés du spectacle de la nature. Fontenelle et Hunauld font appel aux pouvoirs de l'imagination, qui permettront aux lecteurs de voir les processus des principales opérations de la nature, la visualisation tenant lieu d'explication. En outre, le découpage en songes dans le Nouveau Traité rappelle la division des Entretiens en soirées. Le Nouveau Traité appartient donc au genre des entretiens savants, qui ont fleuri pendant le xvIII ${ }^{\mathrm{e}}$ siècle, notamment Les Entretiens physiques d'Ariste et d'Eudoxe de Régnault en 1729, Le Spectacle de la nature ${ }^{18}$ de Pluche et Les Entretiens sur la cause de l'orbite des planètes de Bouguer (1734). Fabrice Chassot ${ }^{19}$ note que le modèle de l'entretien entre maître et disciple dominera dans la seconde moitié du siècle et cite, pour exemple, Le Newtonianisme de M. de Voltaire, ou entretiens d'un étudiant avec un docteur newtonien attribué à Serane (1779), et Les Entretiens sur différentes questions de physique de Le Marchand (1777). On pourrait ajouter à cette liste d'autres ouvrages qui mêlent avec plus ou moins de bonheur les entretiens savants avec la fantaisie de la fiction, voire de la "science-fiction». La forme de l'entretien avec un interlocuteur privilégié sera reprise dans Le Philosophe sans prétention ou l'Homme rare. Ouvrage physique, chymique, politique et moral, dédié aux savans de La Follie (1775). Enfin, le songe est un expédient narratif qu'utilisera Jost de Villeneuve dans Le Voyageur philosophe, prétexte vraisemblable pour des voyages imaginaires qui se situent dans la longue suite de l'Histoire comique des États et Empires de la lune, mais, en dépit de cette ressemblance formelle, l'objet d'Hunauld est de nous transporter dans le corps humain et non chez les habitants de la lune.

\section{Le faux merveilleux}

Le merveilleux du Nouveau Traité relève, dans un premier temps, d'un univers puisé à la fois à la source des contes (le Génie), de la mythologie (Prométhée et Démogorgon), et de la représentation picturale et moralisante à laquelle appartiennent les allégories Chimie, Histoire, Nature, etc. Ce merveilleux évoque tout un pan de la production fictionnelle des $\mathrm{XVII}^{\mathrm{e}}$ et $\mathrm{xVIII}{ }^{\mathrm{e}}$ siècles, souvent critiquée pour son invraisemblance. Ce merveilleux sera rejeté par exemple par Jost de Villeneuve dans Le Voyageur philosophe qui, dans la préface, se

récrie contre ces Génies commodes dont les Romans sont farcis, qui bâtissent en un instant des palais somptueux, qui transforment les pierres en hommes, les hommes en animaux, les animaux en plantes; les Orphées, les Amphions semblent des Etres fabuleux; les hercules, les Achilles, des héros fabriqués par l'imagination pour récréer, ou égarer celle des autres ${ }^{20}$.

Il faudrait, cependant, être un lecteur bien naïf pour se laisser prendre à ces fictions. Ce merveilleux peut être apprécié pour les réminiscences littéraires qu'il suscite, mais il ne troublera pas le lecteur dans l'exercice de sa raison. Les formes en sont surannées, éminemment reconnaissables; elles ont perdu leur pouvoir d'égarement. De fait, dans le Nouveau traité d'Hunauld, ce merveilleux sert d'ornement. La première fonction dont il est investi est d'asseoir l'autorité des intervenants dans leur relation pédagogique avec le disciple. La seconde est de poser un décor aux lignes maitrisées, une nature 
domptée, taillée par la main humaine, illustrant l'adage cartésien de l'homme maître et possesseur de la nature. Hunauld désamorce d'emblée une rivalité éventuelle entre fiction et science. C'est un merveilleux raisonné, une «belle nature » qu'il dessine selon les principes de la science. Les arbres y sont taillés en «berceau », des labyrinthes sont aménagés dans le parc, un lit de gazon s'offre aux conférents sous le feuillage d'un arbre : tout y respire l'ordre et la maîtrise, image inversée de la forêt dans la fiction, lieu de la violence (la chasse), de la solitude et de l'oubli de soi. Hunauld invite son lecteur à se prêter à une lecture allégorique ${ }^{21}$ du merveilleux de la fable. Quand Asclépiade rencontre Prométhée dans le quatrième songe, le Génie le met en garde contre une lecture littérale du mythe. Prométhée n'a pas créé l'homme avec de la boue et le feu qu'il avait dérobé aux dieux, explique le Génie, mais

en son temps les hommes mal disciplinés vivaient dans de si étranges désordres, qu'ils pouvaient bien passer pour n'être que boue méprisable. Il les sut corriger par ses excellentes leçons; et, ranimant ainsi leurs esprits, assoupis dans les ténèbres des sens, par les rayons de cette souveraine sagesse, qu'il leur inspira; il en fit des hommes si différents, qu'il passe pour le réparateur du genre humain ${ }^{22}$.

Ce «faux merveilleux » s'avère donc bien inoffensif, puisqu'il s'annonce comme tel, avec son cortège d'allégories et de souvenirs romanesques. Certes, le merveilleux de la fable prête le flanc à la critique des philosophes qui, selon une lecture quelque peu superficielle, l'accusent d'être le terreau de l'enthousiasme, de la superstition et de l'imagination, ce que Lorraine Daston désigne plaisamment de "unholy Trinity ${ }^{23}$ ". Quand il compose le Nouveau Traité, Hunauld a peut-être conscience de marcher sur un terrain qui se dérobe, tant il s'attache à désamorcer le pouvoir de ce merveilleux compassé. Ses personnages allégoriques et fabuleux ont peu d'éclat; ils embellissent le cadre de la science comme un frontispice peut orner un traité de physique ou de médecine.

\section{La science sensible du médecin}

La médecine est en effet au cœur du projet de Hunauld. S'il a repris le modèle de l'entretien savant entre un maitre et un disciple, c'est qu'il entend toujours se poser aux yeux du lecteur comme médecin. Étant étudiant, Asclépiade a besoin de l'enseignement qui lui est imparti par le Génie et consorts. En outre, Hunauld entremêle à ce premier prétexte un second, qui servira à justifier l'expédient narratif du songe: la maladie d'Asclépiade. Le jeune homme est en effet "rêveur et mélancolique ${ }^{24}$ » et recherche la solitude. "La profonde mélancolie, dans laquelle ils m'avoient trouvé, ne me quittait pas ; elle était même si forte, que bientôt elle me fit perdre la piste et l'idée de la chasse. Accablé par mes rêves et nullement maître de moimême, je fus contraint, dit Asclépiade, de mettre pied à terre $[. . .]^{25}$." Les médecins reconnaîtront en lui les symptômes comportementaux du tempérament mélancolique. Antoine Le Camus attribue leur mal à la «nature grossière des molécules du sang ${ }^{26}$ ». Les molécules devant lutter pour passer dans les vaisseaux sanguins, l'organisme est épuisé par cette lutte interne ; «les organes sont continuellement ébranlés par des mouvements contraires à l'intégrité de l'œeconomie animale. L'âme par son étroite liaison avec le corps, doit concevoir une vraie tristesse, être inquiète, et craindre sa dissociation ${ }^{27}$ ». L'instabilité du sang se communique au corps et à l'âme dans le diagnostic de mélancolie "vagabonde ", car ceux qui souffrent de ce mal «errent sans savoir où ils vont "; ils " aiment la solitude ", et "se retirent dans des lieux solitaires pour n'être point troublés dans la spéculation des objets gigantesques et imaginaires 
dont leur esprit se repaît $»^{28}$. Ainsi, le Génie compare Asclépiade à " un vaisseau, qui, sans pilote, vogue au gré des flots, et qui va se briser contre quelque rocher ${ }^{29} »$. Le médecin anglais James recommande des "céphaliques humectants» et surtout le «sommeil $»^{30}$, remède que Hunauld procure à son héros avec des pavots. À ces considérations sur la mélancolie et sur les soins que ce désordre exige, s'ajoutent des allusions antiques à la profession de médecin. En dénommant son héros Esculape, Hunauld renvoie son lecteur aux pratiques anciennes de l'incubation dans le temple d'Esculape ${ }^{31}$. Le rite consistait à se coucher et à attendre que la divinité vienne visiter les patients endormis pour leur délivrer un message. L'auteur apporte donc une justification tirée de son art et des sources anciennes les plus prestigieuses ${ }^{32}$. Le rêve engage une thérapeutique et réalise le programme annoncé dans le sous-titre: Méditations, et songes sur tous les corps dont la Médecine tire les plus grands avantages pour guérir le corps humain. Hunauld défend l'artifice du rêve, en prenant appui sur les opinions des anciens. Le rêve n'est pas antinomique à la raison, argue-t-il, et il propose de voir dans son ouvrage des "songes raisonnés ${ }^{33}$ ». Tout comme son modèle fontenellien, Hunauld circonscrit par conséquent l'aire du merveilleux dans son ouvrage et le justifie par des motifs vraisemblables, par un «fondement réel ${ }^{34}$ ». L'auteur ne semble pas craindre le pouvoir trompeur de l'imagination, car il met toute sa confiance dans l'expérience sensible du sujet.

5 Certes, le lecteur trouvera bien sous la plume d'Hunauld, par l'intermédiaire du Génie, quelques mises en garde sur les mensonges de l'imagination, mais le plus souvent l'auteur attribue à l'imagination une faculté cognitive attachée aux choses sensibles, qui les déploie dans l'espace, les déplace, et en révèle les actions qui demeurent invisibles. Le Génie recommande, comme préalable à la formation médicale, de s'appuyer sur le témoignage des sens, au motif qu'ils parlent les « langages établis par la Nature même ${ }^{35}$ ». Au médecin de recueillir les signes, les symptômes, les caractères laissés dans la nature et de les rapporter à un système herméneutique. La science empirique et probabiliste du médecin n'est valable que dans le cadre interprétatif mis en place par la tradition et le savoir établi, ce qu'Hunauld désigne par l'«utile»: «Leurs mensonges autorisés par la Nature ne vous déroberont rien de tout ce qui vous est utile ${ }^{36}$. $)$ 'art du médecin est de l'ordre du pratique, du sensible et de l'application. Les sens demeurent l'outil le plus fiable pour le médecin, l'observation étant vérifiée par l'invariabilité du phénomène: "Profitez des effets auxquels vous êtes sensible. C'est assez qu'ils soient constants, invariables; pour qu'ils vaillent autant pour vous que la réalité ${ }^{37}$. » Hunauld est cohérent dans sa démarche puisque, dans les songes suivants, il développera des images sensibles pour permettre de visualiser des processus invisibles. En somme, il continue le roman de la matière que la nature a commencé, lorsque son Génie recommande d'utiliser des "exemples sensibles pour rapprocher de vos sens ces idées trop abstraites, dont votre imagination pourrait se rebuter $^{38}$ ". Le médecin prend donc ses distances avec la méfiance cartésienne envers la connaissance sensible. Curieusement, Hunauld trouvera dans l'analogisme des images plus conformes à l'expérience et au savoir du médecin. Il n'en conserve pas moins le modèle de la machine.

\section{La machine : merveille ou cadavre?}

Le mécanisme se présente comme un système explicatif qui en appelle à la vue de l'esprit pour suppléer aux déficiences des sens: «Un charme secret vous dérobe 
l'artifice. Ce ne sont de toutes parts que tourbillons de poussière diversement agités, que cordages, que poulies, que leviers, en un mot, que machines différentes ${ }^{39}$. " La pensée mécaniste repose sur une analogie première, selon laquelle le monde et tous les êtres qui le composent sont des machines, dont le fonctionnement stable et répétitif est assuré par des processus identiques à ceux qui régissent les machines artificielles. En outre, la compréhension mécaniste de la nature suppose au préalable de faire abstraction des différences accidentelles, et aboutit à une réduction générale à quelques principes de base, de sorte que le fonctionnement d'une entité est transposable aux autres éléments, conformément au principe de l'uniformité des procédés de la nature. Confiant dans les capacités étiologiques du mécanisme, Hunauld parsème son ouvrage d'images qui renvoient à la machine. Il sollicite le modèle mécanique pour expliquer la combustion, les réactions entre éléments chimiques, la croissance des plantes, la digestion et la circulation du sang dans le corps. Ce qui fait dire à Chimie qu' ' autant qu'un habile machiniste a besoin de changer en mille dispositions différentes ses leviers, ses roues, ses poulies, et de varier en autant de manières les déterminations de ses points d'appui et de direction, je suis obligé [sic] de diversifier la forme de mes vaisseaux ${ }^{40} »$. Hunauld exploite l'image bien connue du système hydraulique d'un jardin pour expliquer que la forme n'affecte pas la composition matérielle des corps. Les semences des plantes sont comparées à des « ajutages» de fontaines qui « reçoivent des déterminations d'autant plus différentes, que leurs eaux sont plus rapidement poussées ${ }^{41}$. Ailleurs, le récit actualise l'ambition de l'explication mécaniste. Sur un signe du Génie, tous les arbres deviennent transparents, de sorte qu'Asclépiade voit la sève monter dans les arbres :

Quel prodige, quand j'y pense! ce fut bien dans ce moment que j'appris, à n'en pouvoir plus douter, que la sève circule dans les arbres, et dans les plantes. Je la voyais couler le long des fibres ligneuses dans des canaux qui s'élevaient autour en forme de spirales, petites vis d'Archimède, où la liqueur roulant toujours vers sa pente naturelle, en éludait cependant la détermination. Cette sève se répandait ainsi de côtés et d'autres dans les écorces, où, arrêtées par mille et mille petits vaisseaux entortillés à la manière des corps glanduleux qu'on observe dans les animaux, elle prenait visiblement de nouvelles consistances. Là, elle s'épaississait ; ici, elle se colorait, ou d'un verdâtre clair, ou d'un jaune foncé, ou en un mot de plusieurs autres couleurs; de là elle continuait son cours par mille et mille autres petits canaux dans les feuilles ${ }^{42}$.

L'explication mécaniste est l'élément déclencheur du merveilleux dans le récit, qui dévoile les rouages derrière les effets et met devant les yeux d'Asclépiade le résultat obtenu par la réduction que la raison opère sur les données de l'observation.

6 Toutefois, ce passage rend manifeste le malaise du médecin dans l'usage du modèle mécanique qu'il a pourtant mobilisé. Pour expliquer la montée de la sève, Hunauld suppose des fibres semblables à des vis d'Archimède. Néanmoins, pour suppléer à l'action limitée de la machine, il ajoute des "corps glanduleux» qui modifient «visiblement», mais par un procédé qui demeure mystérieux, la couleur et la consistance de la sève. Les glandes, qu'Hunauld emprunte aux animaux et dont il présume la présence dans la plante, sont révélatrices du besoin qu'il ressent d'ajouter des organes chimiques distincts, là où l'observation ne révèle que des fibres. Le médecin envisage, d'une part, le corps végétal ou humain comme un ensemble vivant et complexe; d'autre part, les outils mécanistes dont il dispose ne lui permettent de décrire cette machine qu'en la démontant en ses parties. L'écriture médicale représente en ce sens une gageure dont Sophie Vasset rend bien compte : « Décrire le vivant, c'est 
essayer de décortiquer la machine du corps alors qu'elle est encore en fonctionnement, tenter de rendre la peau transparente pour voir au travers les mouvements des fluides qui traversent le corps ${ }^{43}$.»

On peut donc déceler chez Hunauld un tiraillement entre une reprise générale et relativement fidèle de l'explication mécaniste du fonctionnement des corps et un rejet de la décomposition, que suscite son attachement aux conceptions de l'hylozoïsme. La science chimique qu'il répand dans son traité lui permet de nuancer le mécanisme et d'y mêler des considérations intermédiaires entre les corps et le spirituel. Le Génie reconnaît les limites de l'explication mécanique des processus de constitution du vivant en distinguant deux sortes d'action mécanique. Il y a, certes, des éléments qui produisent des effets par les lois de la mécanique, déclare-t-il, mais il y en a d'autres, dont les effets ne peuvent se réduire à ces lois ${ }^{44}$. Tels sont les esprits lumineux des semences des choses qui génèrent l'âme végétative dans les plantes, de sorte que l'être vivant comporte un supplément que l'on ne peut ni quantifier, ni observer, ni réduire aux lois de la mécanique. Les machines, déclare le Génie dans le quatrième et dernier songe, sont des cadavres. D'où la nécessité, défend-il, d'accepter l'hypothèse des esprits séminaux qui animent les machines: "Par elles-mêmes cadavres affreux, elles [les machines] ne tiennent de vie, de force, de beauté, que par les esprits seuls; ils en font le merveilleux, et ce n'est que pour faire valoir leurs merveilleuses propriétés que ces machines leur sont préparées ${ }^{45}$. » Pour le médecin, la seule véritable merveille, c'est la vie. La médecine étudie le "corps vivant ", et non le "corps mort ", qu'il laisse aux anatomistes ${ }^{46}$. Le Génie annonce le programme de son enseignement, en distinguant clairement l'objet de sa discipline de celui de l'anatomie :

Je vous ai préparé aux découvertes que vous pourrez faire du côté de son esprit; c'est à présent de son corps qu'il s'agit. Je veux dire de son corps vivant, et non pas de ce cadavre, partie de lui-même si petite, dont l'Anatomie vous pourrait faire la description. La médecine la pourrait négliger, si ce n'était qu'elle a besoin de connaître les organes qui servent à la vie dont elle fait son principal objet ${ }^{47}$.

Outre que la vie est une "puissance qu'on peut dire indifférente au bien ou au mal ", déclaration qui rappelle la conception stoïcienne des adiaphora ${ }^{48}$, Hunauld ajoute que "la vie est véritablement ce qu'il y a de plus considérable dans la machine humaine " qui dépend «de tant de choses différentes, les unes si grandes et si éloignées de vous; les autres si petites, même si peu sensibles, et toutefois si voisines, que vous aurez peine à les rassembler $»^{49}$. Le savoir médical, qui est connaissance de la vie, est difficilement quantifiable et localisable. Si le mécanicien peut sans peine désassembler la machine, il ne parvient toutefois pas à la rassembler. Pour Hunauld, qui se rapproche des préoccupations vitalistes, la vie émane d'une synthèse que l'esprit humain appréhende difficilement dans sa totalité.

Pour graver plus profondément cette différence fondamentale entre la science du vivant et la dissection dans la mémoire de son lecteur, Hunauld crée le type caricatural de l'anatomiste, toujours représenté le «couteau à la main ${ }^{50}$ ». La première critique qu'Hunauld fait aux anatomistes est de vouloir comprendre le corps en ses parties et non comme un tout. L'auteur n'abandonne jamais tout à fait l'analogie du corps comme machine, mais il tempère son système mécaniste. La vie est une faculté de la totalité et ne peut se déduire de l'examen des parties. Il faut, déclare-t-il, comprendre la "mécanique générale» et non les "ressorts particuliers " ${ }^{51}$. Les anatomistes, qui décomposent le corps et tentent de comprendre le fonctionnement général d'après l'analyse des membres, ne sauraient, pense Hunauld, parvenir à une quelconque 
compréhension du corps vivant. La fonction de chaque organe étant déterminée par l'économie intérieure du corps, elle ne peut être déduite de sa morphologie particulière.

Je ne le [développer les mystères] ferai pas à la manière de vos Anatomistes, qui, le couteau à la main, ne cherchent qu'à découper des parties, qui prétendent trouver un Cerveau, un Cœur, un Foie, une Rate, ou plutôt, qui, avant que d'avoir aucune connaissance de ces parties cachées, fouillent à l'aventure, et qui les trouvent çà et là bizarrement placées, sans en savoir la raison; mais qui la cherchent après les avoir découvertes; comme si ce qui parait de leurs formes extérieures ou de leur situations avait quelques rapports avec leur conformation intérieure, et leurs usages ${ }^{52}$.

Face à cette impasse, Hunauld opte pour des concepts et images de la chimie ancienne, sans toutefois renier le mécanisme cartésien dont il a fait la fondation de sa science médicale. La chimie conserve, dans la première moitié du xviII ${ }^{\mathrm{e}}$ siècle, nombre d'éléments irrationnels, que la physique cartésienne n'a pas réduits à des processus mécaniques quantifiables. Science occulte, elle demeure mystérieuse, car la décomposition du mouvement dans les réactions chimiques n'est pas observable. Le Génie avertit son disciple de cette gradation entre le visible, l'imaginaire sensible et les chimères de l'imagination dont les visions ne sont plus arrimées au réel: "Vouloir pénétrer au-delà, il serait inutile. Outre que vos sens vous abandonneraient dans une recherche si téméraire, votre imagination même, toute ingénieuse qu'elle soit à vous amuser de ses fantômes, d'abord que la réalité lui manque, ne trouverait rien dans cet abyme de quoi les former ${ }^{53}$. » L'univers des opérations chimiques, parce qu'il échappe à l'observation, recourt à des représentations du principe de vie, puisées dans le répertoire de l'imaginaire alchimique. Dans cette fabrique d'images, les plantes jouent un rôle important ${ }^{54}$, parce qu'elles figurent, au moins depuis les travaux de Mariotte ${ }^{55}$, comme les chimistes de la nature, et les intermédiaires entre l'homme et son environnement. Elles servent de fixateurs pour représenter des processus chimiques mystérieux. Dans les pages qui suivent, nous examinerons ainsi comment la chimie s'accommode d'un monde dont la plénitude et la diversité des éléments, qui s'interpénètrent, matérialisent la porosité entre les trois règnes de la nature, et comment ces combinaisons autorisent des analogies entre le vivant et l'inanimé.

\section{Les plantes chimistes}

Les éléments chimiques se combinent et « deviennent mille et mille choses différentes ${ }^{56}$ ». Hunauld emprunte sans doute à Gassendi et à la tradition atomiste la conception corpusculaire des éléments premiers qui se mélangent pour constituer toutes les choses de la nature dans des assortiments toujours variés. Hunauld distingue trois éléments de base, sels, souffres, et eau, qui forment une tria prima différente de celle de Paracelse et de Stahl (Soufre, Mercure, Sel), et qui constituent l'ensemble du monde. À partir de là, il adopte une classification où tous les corps chimiques sont classés selon ces trois genres. À l'intérieur de chacun de ces trois groupes, les variétés de ces éléments sont rangées du plus volatil au plus fixe. Enfin, les combinaisons de ces trois éléments sont illimitées ${ }^{57}$. Les molécules de matière s'assemblent selon des mouvements « d'amour ou de haine, d'union ou de désunion" "ou bien que cela soit mécaniquement conduit selon les règles ${ }^{58}$ ", sans qu'Hunauld ne veuille entrer plus en détail sur de possibles facultés de la matière, et qu'il se prononce sur les affinités de la chimie newtonienne ${ }^{59}$, ou sur le mécanisme cartésien. Tout est en circulation, reprend le Génie, il y a un 
"mouvement général par lequel la matière commune est préparée ${ }^{60}$ ». La terre est imbibée de sucs (salins, aqueux, sulfureux) qui se combinent et se cristallisent pour former divers terroirs. Les mouvements et combinaisons sont facilités par la nature poreuse des choses. Ainsi dira-t-il que «la terre n'est pas autre chose qu'une sorte d'éponge ${ }^{61}$ ", « composée d'une infinité d'éponges " ${ }^{62}$. La même métaphore est utilisée pour la terre, pénétrée par les atomes ignés des rayons du soleil ${ }^{63}$ : «En sorte que nageant dans cet immense liquide, c'est comme une éponge jetée dans l'Océan. Oui, je puis vous en assurer très certainement, le corps humain n'est pas plus exactement pénétré dans toute son étendue par les esprits, qui animent, et entretiennent sa chaleur, que la terre l'est par les rayons du soleil ${ }^{64}$.» Ce passage montre que l'analogie, fondée sur la porosité matérielle des choses, habille la matière inerte du costume du vivant. Auparavant éponge, la Terre prend ainsi les traits d'un poisson qui «nage ", "dans l'océan ». Ensuite, la même image est appliquée à un nouvel objet, le corps humain. Par ce procédé, Hunauld mêle l'océan et le soleil, l'éponge et le corps humain pour peindre un même processus d'interpénétration. Ces mouvements de permutation participent à la création d'un tissu analogique de correspondances entre les objets reliés entre eux par des comparants qui font fi des distinctions entre l'animé et l'inanimé. Hunauld renforce ces associations par le présupposé physique que le monde est un tout dont les éléments constitutifs sont soumis aux mêmes lois mécaniques. L'énoncé hippocratique du "commerce perpétuel ${ }^{65}$ ", qui régit les échanges entre les organes du corps humain, s'applique à la terre, au soleil, à l'air et à tous les éléments. De ces analogies fonctionnelles dont les termes sont interchangeables, il résulte un maillage extrêmement dense du monde: «[...] dans cette vaste machine de l'Univers tout est lié par des relations si exactes, qu'elles ne forment, à proprement parler, toutes ensemble que comme un corps continu ${ }^{66}$." Dans un tel contexte, l'ambivalence d'Hunauld vis-à-vis de l'anatomie prend un relief nouveau. Vouloir disséquer la nature, c'est la scinder en ses parties, et parce que la trame des processus naturels relie les objets les uns aux autres dans une myriade d'interactions, c'est en empêcher la connaissance. Le principe cartésien de la divisibilité de la matière entre en contradiction avec celui de l'intégrité du corps, condition nécessaire à la vie.

8 En outre, Hunauld s'appuie sur l'universalité d'une matière commune qu'il doit sans doute à Paracelse, pour qui les corps des vivants sont constitués des mêmes éléments que celui des métaux et minéraux. C'est pour cette raison que les médecins paracelsiens et Van Helmont administraient à leurs patients des médicaments issus de substances minérales et non uniquement végétales ou animales, comme le recommandaient les médecins galénistes ${ }^{67}$. De sorte que, la matière étant commune et les procédés équivalents, les analogies glissent sans heurt de la terre au corps humain, et du corps humain aux plantes. L'analogie n'est pas ici seulement pourvue d'une fonction scénographique et pédagogique; elle exprime une identité fonctionnelle, qui donne la liberté d'établir des équivalences, dans un mélange des genres qui évoque la théorie médiévale des signatures. Dire, comme le fait Hunauld, que la terre est un corps, signifie qu'elle est composée d'éléments qui effectuent activement ou passivement les mêmes fonctions que les substances dans les organes d'un corps, et que, alors que les organes de la terre n'ont pas encore été observés, on doit les déduire des produits de leur organisation: "Je vous ai dit que la terre avait comme ses entrailles, et ses viscères, ses philtres, ses colatoires ${ }^{68}$. Je dirais même quasi comme son Foie, sa Rate, ses Poumons, et les autres parties destinées à la préparation des sucs alimentaires. Elle a aussi ses os, comme un squelette très régulièrement formé ${ }^{69}$. " Ainsi, la terre a un 
squelette, tout comme le corps humain, qu'Hunauld comparera ensuite à la charpente d'un édifice parce que telle est sa fonction. Les similarités peuvent donner lieu à un véritable feu d'artifice d'images tirées de l'expérience sensible du vivant et liées entre elles par des homologies fonctionnelles.

\section{L'arbre-animal}

Les deux processus fondamentaux du vivant que le médecin Hunauld retient dans sa description du corps humain sont la digestion et la circulation du sang. La vie tient de deux organes, l'estomac et le réseau sanguin, sièges respectifs de deux opérations de transformations chimiques, la fermentation et la combustion. L'auteur compare le vivant à un arbre dont le tronc se divise en deux branches. La fermentation est le processus chimique de la digestion, elle-même étape essentielle de la nutrition, faculté des végétaux, que l'on nomme "âme végétative » qui relie les êtres vivants à la terre. Quant à la combustion, elle est l'opération de l'animalité, véhiculée par le sang, dont la chaleur vient des atomes ignés. De là, l'instituteur conclut : "Voilà tout l'homme. Son système général ne roule que sur cette très simple œconomie. La branche du suc nourricier s'étend le long du genre végétal ; l'autre branche qui anime, constitue avec ses vaisseaux, et les organes de son ressort, le genre animal ${ }^{70}$. " Cette représentation, qui se démarque de la pensée de Descartes ${ }^{71}$, continue la tradition aristotélicienne, qui faisait des facultés une matrice fonctionnelle, les besoins suscitant l'existence des organes destinés à les satisfaire. L'être humain doit la vie à la faculté végétative. " Les esprits végétaux, ou naturels, entretiennent la vie; les animaux la décorent, la perfectionnent, par les sensations différentes, qui vous distinguent des Arbres, et des Plantes ${ }^{72}$. » L'homme est un arbre amélioré : « Ainsi, je conçois déjà l'homme, à sa figure près, comme un arbre capable de se remuer, et je comprends que c'est l'animal ajouté au végétal, qui fait remuer cet arbre, et qui le rend pareillement susceptible de tous ces sentiments de lumière, de couleurs, de saveurs, d'odeurs, de sons, de toucher, que l'homme partage avec les autres animaux ${ }^{73}$." La faculté végétative, ou «âme végétante ", confondra ainsi le corps humain avec la plante et, par un raccourci poétique, l'homme est non seulement arbre, mais il est arbre avant d'être animal. L'âme végétative lui procure nutrition et croissance, qui sont, dans l'optique du médecin Hunauld, les fonctions primordiales du vivant. En effet, il accorde clairement la prééminence à la faculté végétative. Dans le discours de Démogorgon, le corps humain se réduit à une construction dont la base est fournie par les organes assurant la faculté végétative (croissance et nutrition). Le reste de l'édifice est consacré aux besoins particuliers de l'animalité, dont fait partie la génération. En somme, l'animal " postiche », est greffé sur le végétal, qui lui donne la vie:

Mais dans ce parallèle de l'un et de l'autre, il ne faut pas confondre certains organes des Animaux, qui ne leur ont été ajoutés qu'à raison de certaines propriétés singulières, par lesquelles ils doivent être distingués du reste des choses. Ils ont dû marcher, voir, entendre, flairer, goûter, parler, et se reproduire par les voies destinées à la propagation de leurs espèces. Tout ce postiche est ajouté à leur nature indépendamment de cette vie qui les anime, et de ces facultés qu'ils ont pour se nourrir des aliments qu'ils ont digérés, et qui circulent dans leurs veines; ou plutôt ces organes profitent de ces facultés, et de cette vie, comme de surérogations qui servent à la perfectionner par l'excellence des usages auxquels ils la font servir $^{74}$. 
Les parties et fonctions animales sont donc un supplément, des "parties accessoires ${ }^{75}$ ». Alors que les plantes tirent leurs aliments de la terre, le corps nécessite des organes pour dupliquer les conditions matérielles dans lesquelles l'arbre se nourrit. Ces premières analogies tirées d'une longue tradition aristotélicienne suscitent des images de rapport entre les règnes.

Examinons, par exemple, comment Hunauld, par l'intermédiaire de Prométhée, représente l'estomac, qui est, selon lui, le siège de la vie dans le corps humain. Le médecin rappelle que l'estomac transforme les matériaux bruts ou secondaires pour les adapter à la physiologie humaine; il équivaut, à ce titre, à la terre des arbres.

Mais ce qui augmente beaucoup le nombre de ces parties nourricières dans les Animaux, vient de ce que n'étant pas, comme les Arbres et les Plantes, attachés à la terre pour puiser immédiatement dans son sein le suc nourricier, qui devait être façonné dans leurs organes, il était nécessaire d'ajouter dans le centre de leur machine des parties propres à contenir des matières, qui, travaillées à propos, pussent y tenir lieu de cette terre féconde, d'où la nourriture se pouvait puiser ${ }^{76}$.

Hunauld assimile donc l'estomac à une caisse remplie de terre d'où croît un arbre, image qu'il développe longuement, et qui n'a rien de vraisemblable :

[V]oilà cette grande caisse portative, et, pour l'être avec moins d'embarras, située dans le centre de la machine de l'animal, où sont contenus les aliments, qui, au moyen de leur dissolution, deviennent une terre féconde, pleine de suc, insolée par les continuelles irradiations des esprits, enfin continuellement remuée ; non par les mains d'un Jardinier habile, mais par le moyen de ces mouvements péristaltiques, ou vermiculaires, dont les intestins sont continuellement agités ${ }^{77}$.

L'image est frappante, à double titre. Elle a pour fin d'imprimer la fonction de l'estomac dans l'imaginaire de l'étudiant en médecine qu'est Asclépiade, et de tisser une série de permutations analogiques d'Andrea Cesalpino, pour qui les racines servent de bouche et d'estomac aux plantes ${ }^{78}$. L'estomac, en étant le centre de la faculté nutritive, relie les hommes aux plantes. Le corps humain est figuré par un arbre planté dans une caisse au niveau de l'estomac. Pour la raison que la digestion raffine, transforme les matériaux en matière subtile, elle peut être figurée par la terre qui entoure les racines d'un arbre de sorte que Hunauld se permet de réduire l'homme à l'arbre : «Imaginez-vous donc que dans les Animaux, l'estomac est tout entier du genre végétal; qu'il fait avec les entrailles, et toutes leurs dépendances, partie des racines de l'Arbre animal ${ }^{79}$. » Dans le discours d'Hunauld, les permutations donnent lieu à des quasi-métamorphoses. Dans le passage cité ci-dessus, la comparaison fonctionnelle entre l'estomac et la terre fait de la végétalité une propriété, de sorte que le corps humain fait plus que contenir un arbre en caisse, il est arbre. Cohérent dans sa démarche homologique, Hunauld recommandera ainsi au futur médecin de «cultiver " l'estomac comme on cultiverait une plantation: «Vous cultiverez leur estomac comme le lieu où l'arbre est planté ; vous y procurerez une bonne dissolution des aliments, accommodée à leurs espèces ${ }^{80}$. »

\section{Le merveilleux végétal}

Or Hunauld va plus loin que ses prédécesseurs dans la végétalisation ${ }^{81}$ du corps humain. Parce qu'à la suite de Van Helmont, il voit, dans l'estomac et les opérations de la digestion, le siège et les conditions fondamentales de la vie, Hunauld développe longuement la vertu germinative et la force séminale des plantes, "source immédiate des principales facultés des arbres et des plantes, principe de leur vie végétante ${ }^{82} »$ : «[...] c'est aussi dans ces racines animales, qu'est implanté l'esprit vital, qui anime tout 
ce qui est de son espèce, ou plutôt tout l'animal ; puisque le genre végétal en compose la plus grande partie ${ }^{83}$.» Même si Hunauld adopte l'image cartésienne de la flamme pour la seconde opération du vivant - la combustion - dans le sang qui charrie les atomes ignés du soleil, le médecin ne fait pas du circuit sanguin le siège de la sensibilité, qu'il situe dans l'estomac. "C'est de là, que l'estomac est de toutes les parties du corps humain la plus sensible, et comme la clef principale de la voûte, dans ce grand édifice ${ }^{84}$.» Tandis que le cœur, selon Hunauld, n'est qu'une pompe assurant la circulation du sang, l'estomac est le " principe de tous les mouvements vitaux ${ }^{85}$ ».

10 L'image de l'estomac en caisse de jardinage n'est de pas de l'ordre du représentable selon les règles $d u$ mécanisme et $d u$ rationalisme. Hunauld sollicite une autre perception du vivant, fondée en partie sur l'expérience sensible du médecin et ramenée à des rapports analogiques selon lesquels le corps humain est réductible à deux fonctions physiologiques premières, la combustion et la fermentation. Chacune de ces opérations ont leur siège (cœur et estomac), leur principe chimique (souffre et humide radical), leur âme (animale et végétative), leur faculté (sensibilité et croissance), et enfin leur référent macrocosmique (soleil et terre). Descartes avait nié toute faculté à la matière, avait exclu les forces occultes de son système physique mécanique. Hunauld les réintroduit à la faveur de la chimie, dont les opérations fondamentales de la fermentation et de la combustion animent le monde et désagrègent la barrière entre l'animé et l'inanimé. Pour Hunauld, le monde est un grand animal ${ }^{86}$. On pourrait le croire adepte du pansensisme de Campanella ${ }^{87}$, mais il s'en défend. Il se contente de soutenir que le monde est composé de parties «organiques ${ }^{88}$ », dont la finalité est de produire ou de détruire. On pourrait le croire vitaliste, mais nulle part Hunauld n'attribue de sensibilité aux fibres.

11 Les analogies et métaphores, qui tendent à brouiller les distinctions entre les trois règnes de la nature, sont pour Hunauld le matériel principal d'un merveilleux qu'il décèle dans les opérations de la chimie. De là, son intérêt pour les organes chimiques - estomac, glandes -, plutôt que mécaniques - cœur, muscles. Il infléchit les comparaisons mécanistes des parties du corps par des analogies non mécanistes, car le spectacle ne montre pas les différentes étapes d'un mouvement saisi dans sa successivité depuis l'immobilité première jusqu'à la complétion du geste. Les voies de la chimie sont impénétrables et requièrent un saut de l'imaginaire, entre un pendant et l'autre de l'analogie, dont la logique fonctionnelle et discursive est assurée par les images végétales. Les plantes n'étant pas dotées d'organes identifiables, les opérations $\mathrm{du}$ vivant s'effectuent sans que l'on sache, à l'époque d'Hunauld, comment les différentes parties de la plante contribuent aux transformations de matière exigées par les processus de la croissance et de la reproduction. En un sens, la plante représente l'objet impossible à anatomiser. Disséquer un arbre, une branche, une feuille permettait, au mieux, de suivre des faisceaux de fibres et de distinguer certains canaux, mais le caractère indistinct des parties internes rendait difficile d'en inférer une fonction physiologique ${ }^{89}$. La circulation de la sève est débattue en $1667^{90}$, elle ne fait toujours pas consensus en $1741^{91}$. Le naturaliste peut donc agir à l'entour de la plante, en mesurant sa consommation d'eau comme le firent Van Helmont ${ }^{92}$ et Boyle, et sa transpiration après les travaux de Hales ${ }^{93}$. La clef pour comprendre Hunauld, c'est donc son rejet de la dissection. On pourrait également soupçonner qu'il abhorrait la distillation pourtant communément pratiquée par les chimistes, non sans susciter de controverse $^{94}$, parce qu'elle détruisait ce qu'elle visait à séparer et rendre visible, mais 
Hunauld n'en dit mot. C'est dans la déconstruction que réside le merveilleux du mécanisme, dans le dévoilement d'un montage simple, dont les effets apparents sont complexes. Or, avec le refus de la dissection, le merveilleux mécanique perd de sa valeur démonstrative. Il peine à donner une représentation de processus physiologiques plus complexes, des viscères et des organes chimiques dont l'estomac est l'exemple emblématique, mais auquel on pourrait substituer le foie ou les poumons. Interviennent alors les plantes pour soutenir des images de procédés de transformation chimique, car elles sont entendues, dans cette première moitié du xvIII siècle, essentiellement comme des tubes vaporisants qui assurent la transformation entre les états liquide et gazeux de l'eau. À l'instar des corps glanduleux, les images végétales rendent donc sensibles des opérations chimiques, tout en occultant les étapes intermédiaires entre la substance initiale et le produit qui en résulte. L'analogisme de la chimie alchimique vient donc combler les lacunes laissées par l'explication mécaniste des choses. Il n'y a donc pas lieu d'opposer un imaginaire mécaniste à celui de la chimie ancienne $e^{95}$. Les images d'Hunauld se ressentent de cette double allégeance, à la fois au mécanisme (système hydraulique, vis d'Archimède) et à l'analogisme prédominant dans la chimie traditionnelle (les correspondances entre arbre, estomac, fermentation et humide radical). Avec le mécanisme pour assise, la science chimique et l'imaginaire végétal se rencontrent au seuil du représentable, le végétal assurant la continuité analogique entre le sensible et l'invisible, là où les images du mécanisme, qui reposent sur la contiguïté des éléments (manivelle, poulie, ressort), échouent à représenter des processus d'un autre ordre.

$\mathrm{Au}$ terme de notre enquête, il nous a donc paru que la distinction entre vrai et faux merveilleux s'est avérée inopérante pour saisir les formes de merveilleux que montre l'ouvrage d'Hunauld. Il est vrai que les pouvoirs de la fable sont sévèrement affaiblis dans le Nouveau Traité de physique. Les songes sont occasionnés par des causes vraisemblables et médicales. Les figures allégoriques et mythologiques n'ont d'autre fonction que d'enseigner au jeune Asclépiade les principes des trois règnes. Tout conspire à circonscrire la matière de la fable et à l'assujettir à des impératifs pédagogiques et philosophiques. La feinte de la fiction est réduite au rang d'ornement, ou de dorage de pilule, pour mieux encadrer une autre sorte de merveilleux, tirée des images et du discours de l'explication mécaniste d'une part, et de l'univers chimique d'autre part. Hunauld fait sien l'héritage cartésien qu'il revoit sans doute à la lumière des enseignements de Boerhaave. Néanmoins, le médecin reconnaît dans le mécanisme une difficulté à traduire de manière discursive la force du vivant. De là vient son animosité envers les anatomistes, les chirurgiens, tous ceux qui analysent, décomposent, dissèquent, démembrent. Se tournant vers la chimie et l'analogie universelle, Hunauld propose une vision unitaire de la nature, dont la science permet de connaître l'enchevêtrement des matières, la prolixité des mouvements, l'uniformité des procédés conjuguée à la diversité des entités naturelles (éléments chimiques, végétaux, animaux), ce que le discours rend par des images principalement végétales. On pourrait qualifier ces références à la chimie de passéistes, si une telle attitude critique ne venait gommer la richesse d'un système de compréhension du monde, dans lequel Hunauld vient puiser des modèles explicatifs, des analogies, des images et des principes cosmologiques. Ainsi, plutôt que d'envisager sa démarche comme vestige d'une pensée archaïque, il serait plus juste de voir qu'Hunauld contribue, dans un traité à la fois actuel et inactuel, au mouvement que remarque Nathalie Vuillemin lors de la deuxième moitié du xvIII siècle, quand les philosophes des Lumières sentent le besoin 
de « réanimer » " une nature que géomètres et mathématiciens avaient eu tendance à momifier : à l'image d'une machine toute entière soumise aux lois que lui impose son créateur, se substitue peu à peu celle d'un être agissant et fécond ${ }^{96} \%$. L'exemple d'Hunauld montre donc que l'imaginaire alchimique procure de nouvelles sources de compréhension ${ }^{97}$ de la nature, dont la porosité générale facilite les échanges entre le minéral, le végétal et l'animal, entre l'humain et le non-humain.

\section{NOTES}

1. Voir J.-R. Carré, «La nature sans miracle. Le faux merveilleux et le merveilleux vrai de la nature (astronomie, physique, chimie) », dans La Philosophie de Fontenelle ou le Sourire de la raison [1932], Genève, Slatkine Reprints, 1970, p. 298-319. Voir le «merveilleux vrai » de Réaumur dans J.-Ch. Abramovici, «Du "merveilleux vrai" des "petits animaux". Réaumur, entre rococo et Lumières ", Dix-huitième siècle, no 42, 2010, p. 305-320.

2. N. Vuillemin, Les Beautés de la nature à l'épreuve de l'analyse. Programmes scientifiques et tentations esthétiques dans l'histoire naturelle du XVIII siècle (1744-1805), Paris, PSN, 2009, p. 228.

3. Nous nous limitons volontairement à cet aspect de l'analogie, notion bien trop complexe pour être abordée ici exhaustivement.

4. Encyclopédie, «Analogie (Logique et Gramm.)», ENCCRE - Édition Numérique Collaborative et CRitique de l'Encyclopédie (1751-1772).

5. J. E. Schlanger, Les Métaphores de l'organisme, Paris, Vrin, 1971, p. 26.

6. On peut consulter avec profit l'étude d'A. Gaillard, «Que peuvent les savoirs pour le conte - et le conte pour les savoirs ? ", Féeries, no 6, 2009, p. 9-19.

7. Nous renvoyons le lecteur aux travaux de J. Marx, de Ph. Vincent et d'Y. Citton, et aux analyses publiées dans l'excellent recueil intitulé Imagination scientifique et littérature merveilleuse. Charles Tiphaigne de la Roche, Pessac, PUB, 2014.

8. Compte rendu dans le Journal des sçavans, août 1742, p. 451-464. Le Mercure de France (déc. 1742, $1^{\mathrm{er}}$ vol., p. 2682) se contente d'en annoncer la publication. La Suite de La Clef, ou Journal historique (juill. 1742) en donne un résumé parsemé de remarques élogieuses (p. 165-168), contrairement à Desfontaines (Observations sur les écrits modernes, t. XXIX, lettre ccccxxiii, Paris, 1742, p. 67-68) qui constate que le genre est « usé ».

9. G. Armand, Les fictions à vocation scientifique de Cyrano de Bergerac à Diderot: vers une poétique hybride, Pessac, PUB, 2013.

10. Les deux volumes ont paru au début de l'année 1742. L'enregistrement date du 3 février 1742 et l'approbation du censeur du 29 décembre 1741. Hunauld décède en décembre 1742.

11. Docteur en médecine à Reims en 1725, Hunauld (1701-1742) poursuit des études d'anatomie à Paris avec Bénigne Winslow et Joseph-Guichard Duverney. Membre de l'Académie des sciences en 1724, il devient professeur d'anatomie en 1730. Il est parfois confondu avec son grand-oncle médecin, Pierre Hunauld, auteur d'un ouvrage sur les vapeurs. La Mettrie, qui a suivi son enseignement en anatomie, lui rend hommage dans sa traduction des Institutions de médecine de Mr. Herman Boerhaave, IV, 1747, p. 96, 255, 309, et en particulier 315 : « [...] mon cher et premier maître en anatomie dont la mémoire me sera toujours aussi triste que chère [...]. »

12. Dissertation en forme de lettres, au sujet des ouvrages de l'auteur du livre sur les maladies des os; ou l'on examine plusieurs points de chirurgie \& d'anatomie, à l'occasion du Livre des maladies des os, \& des 
Mémoires que le même auteur a donnés à l'Académie royale des sciences, 1726 ; Le Chirurgien médecin ou Lettre au sujet des chirurgiens qui exercent la médecine; adressée à l'auteur de la Dissertation en forme de lettres, touchant les ouvrages de l'auteur du Livre de la maladie des os, par M.A.R.D.E.M., 1726.

13. Le Baillon ou Réflexions adressées à l'auteur de la Lettre insérée dans le Mercure du mois d'Août dernier, au sujet de la dispute qui s'est élevée entre M. Maloet, et un quidam soi-disant médecin anglais, d'une part et les Chirurgiens de l'autre part, Amsterdam, 1737.

14. J. Rohault, Traité de physique [1671], préface S. Mazauric, Paris, Éditions du Comité des travaux historiques et scientifiques, 2014.

15. Fr.-J. Hunauld, Nouveau Traité de physique, Paris, Didot, 1742, t. I, p. 5.

16. Voir S. Matton, «La figure de Démogorgon dans la littérature alchimique », dans D. Kahn et S. Matton (éds), Alchimie : art, histoire et mythes, Paris-Milan, S.E.H.A./Archè, 1995, p. 265-346.

17. Rappelons avec Fabrice Chassot que « jusqu'en 1751, le modèle fontenellien est le plus imité » (Le Dialogue scientifique au XVIII siècle. Postérité de Fontenelle et vulgarisation des sciences, Paris, Classiques Garnier, 2011, p. 34).

18. L'influence de Pluche est déterminante et devrait faire l'objet d'une analyse que l'espace qui nous est alloué ne nous permet pas de mener ici.

19. F. Chassot, ouvr. cité, p. 36.

20. D. Jost de Villeneuve, Le Voyageur philosophe dans un pais inconnu aux habitans de la Terre de M. de Listonai, Amsterdam, t. I, 1761, p. 3.

21. Ce faisant, Hunauld invite le lecteur à une pratique de lecture déjà ancienne à son époque et qui remonte à la période avant 1660, selon Aurélia Gaillard, où «il s'agissait d'un pacte allégorique - étant entendu que sous ce terme nous plaçons toutes les méthodes et exégèses qui consistaient à lire sous la fable un autre sens, à soulever son voile. Il s'obtenait soit par addition (d'ornements, de commentaires, de paraphrases), soit par soustraction (coupures et censures, fragmentation) - parfois, plus rarement, par complet remaniement ». Fables, Mythes, Contes. L'esthétique de la fable et du fabuleux (1660-1724), Paris, H. Champion, 1996, p. 79.

22. Fr.-J. Hunauld, ouvr. cité, t. II, p. 4.

23. L. Daston et K. Park, Wonders and the Order of Nature 1150-1750, New York, Zone Books, 1998, p. 334.

24. Fr.-J. Hunauld, ouvr. cité, t. I, p. 31.

25. Ibid., p. 4.

26. A. Le Camus, La Médecine de l'esprit, Paris, Ganeau, 1749, p. 156-157.

27. Ibid., p. 157.

28. James, Dictionnaire universel de Médecine, de Chirurgie, d'Anatomie, [...]. Traduit de l'Anglois de M. James par Mrs Diderot, Eidous \& Toussaint, Paris, Briasson-David-Durand, vol. IV, 1747, p. 1214.

29. Fr.-J. Hunauld, ouvr. cité, t. I, p. 9.

30. James, ouvr. cité, p. 1215.

31. A. Taffin, « Comment on rêvait dans les Temples d'Esculape », Bulletin de l'Association Guillaume Budé, vol. 1, no 3, 1960, p. 325-366.

32. Hunauld pouvait invoquer l'autorité d'Hippocrate concernant les rêves. "Les signes qui se montrent dans le sommeil seront trouvés posséder pour toute chose une grande vertu par celui qui saura en juger sainement. » Hippocrate, Du Régime, Livre IV, Des songes. Voir l'ouvrage ancien de K. Sprengel, Histoire de la médecine depuis son origine jusqu'au dix-neuvième siècle, Paris, Deterville, 1815, t. V, p. 388-399.

33. Fr.-J. Hunauld, ouvr. cité, t. I, p. 16.

34. "Je n'ai rien voulu imaginer sur les habitants des mondes, qui fût entièrement impossible et chimérique. J'ai tâché de dire tout ce qu'on en pouvait penser raisonnablement, et les visions même que j'ai ajoutées à cela, ont quelque fondement réel. Le vrai et le faux sont mêlés ici, mais ils y sont toujours aisés à distinguer.» Fontenelle, Entretiens sur la pluralité des mondes, op. cit., p. 140. où ? 
35. Fr.-J. Hunauld, ouvr. cité, t. I, p. 31.

36. Ibid., p. 29-30.

37. Ibid., p. 31.

38. Ibid., p. 35.

39. Ibid., p. 28-29.

40. Ibid., t. II, p. 58.

41. Ibid., t. I, p. 110.

42. Ibid., p. 157.

43. S. Vasset, Décrire, prescrire, guérir. Médecine et fiction dans la Grande-Bretagne du XVIII ${ }^{e}$ siècle, Paris, Hermann, 2013, p. 41.

44. Fr.-J. Hunauld, ouvr. cité, t. I, p. 114.

45. Ibid., p. 159. Nous soulignons.

46. Ibid., p. 22. L'intérêt de Stahl pour le vivant a pu conforter les positions d'Hunauld, mais la relative indifférence de Stahl pour la chimie et son refus du ferment gastrique suffisent pour les différencier. Voir J. R. Partington, A history of chemistry, Londres, Macmillan, 1961, vol. II, p. 655-659.

47. Fr.-J. Hunauld, ouvr. cité, t. I, p. 22. Nous soulignons.

48. Diogène de Laërte, dans son compte rendu de la philosophie des stoïciens, rapporte qu' «au nombre des choses indifférentes, ils placent tout ce qui n'est ni utile ni nuisible; d'une part, la vie, la santé, le plaisir, la beauté, la force, la richesse, la réputation, la noblesse ; de l'autre, la mort, la maladie, la douleur, la laideur, la faiblesse, la pauvreté, une vie sans gloire, une naissance obscure et toutes les choses de ce genre ». Vies et doctrines des philosophes de l'Antiquité, trad. Ch. Zévort, t. I, liv. VII, chap. 1, $2^{\mathrm{e}}$ part., Paris, Charpentier, 1847, p. 100-101. Pour une analyse historique des connaissances des alchimistes en philosophie stoïcienne, nous renvoyons le lecteur à l'étude de S. Matton, "Alchimie et stoïcisme: à propos de récentes recherches ", Chrysopœia, V (1992-1996), p. 5-144.

49. Fr.-J. Hunauld, ouvr. cité, t. I, p. 23.

50. Personnage contre lequel Hunauld avait déjà lancé ses foudres dans ses opuscules polémiques en 1726 et 1737.

51. Fr.-J. Hunauld, ouvr. cité, t. I, p. 18.

52. Fr.-J. Hunauld, ouvr. cité, t. II, p. 18-19.

53. Ibid.t. I, p. 36.

54. Il est notable que Tiphaigne de la Roche porte lui aussi une attention particulière au végétal. Lire à ce sujet la fine analyse d'E. Sempère, "Le végétal chez Tiphaigne, image(s) ou modèle(s) ? ", dans Y. Citton, M. Dubacq et Ph. Vincent (dir.), ouvr. cité, p. 211-230.

55. Les travaux de Mariotte ont porté sur les principes chimiques de plantes dans son Essai sur la végétation des plantes (1676). Voir J. R. Partington, ouvr. cité, 1962, vol. III, « Chemistry in France I. 1600 to $1700 »$, p. 11.

56. Fr.-J. Hunauld, ouvr. cité, t. I, p. 38.

57. Ibid., p. 44.

58. Ibid., p. 77-78.

59. Voir B. Joly, Descartes et la chimie, Paris, Vrin, 2011, p. 218.

60. Fr.-J. Hunauld, ouvr. cité, t. I, p. 106.

61. Ibid., p. 47.

62. Ibid., p. 48.

63. Voir Fr. Bernier, Abrégé de la philosophie de Gassendi, $2^{e}$ éd., t. V, Lyon, Chez Anisson, Posuel \& Rigaud, 1684, p. 98 et suiv.

64. Fr.-J. Hunauld, ouvr. cité, t. I, p. 64-65.

65. Ibid., p. 51.

66. Ibid., p. 128. 
67. Informations tirées de B. Joly, ouvr. cité, p. 23.

68. «Colatorium » désigne toute sorte de passage comparable à un couloir en anatomie selon le Dictionnaire universel de James, ouvr. cité, vol. III, p. 676.

69. Fr.-J. Hunauld, ouvr. cité, t. I, p. 73.

70. Ibid., t. II, p. 41.

71. Vincent Aucante rappelle que «Descartes, comme on le sait, rejette la conception aristotélicienne de l'âme. Selon lui, le corps n'a pas besoin pour exercer ses fonctions vitales et ordinaires d'aucune « âme végétante ou sensitive » (La philosophie médicale de Descartes, Paris, PUF, 2006, p. 40-41).

72. Fr.-J. Hunauld, ouvr. cité, t. II, p. 161.

73. Ibid., p. 10.

74. Ibid., t. I, p. 81-82.

75. Ibid., t. II, p. 22.

76. Ibid., p. 23.

77. Ibid., p. 102-103.

78. A. Cesalpino, De plantis libri XVI, Florence, Giorgio Marescotti, 1583.

79. Fr.-J. Hunauld, ouvr. cité, t. II, p. 73.

80. Ibid., p. 26.

81. Hunauld se démarque de tous ses contemporains qui réduisent le végétal à l'animal par le biais de l'analogie, après la découverte et l'acceptation de la reproduction sexuée des spermatophytes (plantes à graines) modelée sur celle des animaux. Voir G. Canguilhem, «Du singulier et de la singularité en épistémologie biologique", dans les Études d'histoire et de philosophie des sciences [...], $7^{\text {e }}$ éd., Paris, Librairie philosophique J. Vrin, 1994, p. 211-225.

82. Fr.-J. Hunauld, ouvr. cité, t. I, p. 144.

83. Ibid., t. II, p. 74.

84. Ibid., p. 74-75.

85. Ibid., p. 75.

86. "Je pourrais comparer le globe terrestre au corps d'un grand animal. Non qu'il vive comme lui, qu'il soit sensible, et capable de multiplication; mais parce qu'il est régulièrement composé de parties que j'appelle organiques » (Fr.-J. Hunauld, ouvr. cité, t. I, p. 32).

87. Hunauld a pu également s'inspirer de l'ouvrage de Pierre Borel, Discours nouveau prouvant la pluralité des Mondes, que les Astres sont des terres habitées et la terre une étoile, qu'elle est hors du centre du monde dans le troisième ciel, et se tourne devant le Soleil qui est fixe, et autres choses très curieuses (1657), en particulier du chapitre 19: "Par quelles raisons on peut prouver que le monde est animé », p. 26-28.

88. Fr.-J. Hunauld, ouvr. cité, t. I, p. 32-33.

89. En témoignent les hypothèses et tâtonnements de Nehemiah Grew sur la croissance des plantes, la fonction des pétales, du pollen, etc., dans son Anatomie des plantes, qui contient une description exacte de leurs parties et leurs usages, et qui fait voir comment elles se forment, comment elles croissent. Par Mr. Nehemiah Grew, Leide, 1785.

90. R. Heller, "Mariotte et la physiologie végétale», dans Mariotte, savant et philosophe (†1684). Analyse d'une renommée, Paris, Librairie philosophique J. Vrin, 1986, p. 185-203. La circulation de la sève est réfutée par Stephen Hales dans La Statique des végétaux (1727) traduite par Buffon en 1735, p. 10

91. G.-A. Bazin, Observations sur les plantes et leur analogie avec les insectes, Strasbourg, 1741, p. 123.

92. Pierre Le Lorrain de Vallemont relate la célèbre expérience de Van Helmont sur une branche de saule à la fin de laquelle il conclut en affirmant que les végétaux tirent leur matière de l'eau (p. 340). Il décrit également les expériences de Robert Boyle sur un melon des Indes (p. 322 et suiv.) dans les Curiositez de la nature et de l'art sur la végétation ou l'Agriculture et le jardinage dans leur perfection, nouv. éd., Paris, 1709. 
93. S. Hales, ouvr. cité.

94. Voir A. Stroup, A Company of Scientists. Botany, Patronage, and Community in Seventeenth-Century Parisian Royal Academy of Sciences, Berkeley, University of California Press, 1990, notamment le chapitre $7:$ « Justifying the chemical analysis of plants », p. 89-103.

95. Il serait intéressant de mettre l'ouvrage d'Hunauld en regard avec les textes de son élève La Mettrie qui a recours à des thématiques chimiques pour dépasser les limites du modèle mécaniste. Voir à ce propos l'analyse de Fr. Pépin, «La Mettrie et la chimie », dans A. Paschoud et Fr. Pépin (dir.), La Mettrie. Philosophie, science et art d'écrire, Paris, Éditions matériologiques, 2017, p. 57-90.

96. N. Vuillemin, ouvr. cité, p. 264.

97. À propos des modes d'identification entre l'humain et le non-humain, les suggestions de Philippe Descola nous semblent particulièrement suggestives, notamment la distinction entre analogisme et naturalisme, dont Hunauld pourrait représenter une forme hybride. Voir La Composition des mondes. Entretien avec Pierre Charbonnier, Paris, Flammarion, coll. "Champs essais ", 2014.

\section{RÉSUMÉS}

Dans le Nouveau Traité de physique (1742), ouvrage méconnu du médecin François-Joseph Hunauld (1701-1742), l'auteur croise le savoir scientifique avec le merveilleux de la fiction. Inspiré par les Entretiens sur la pluralité des mondes de Fontenelle et du Spectacle de la Nature de Pluche, Hunauld présente un voyage imaginaire lors duquel des figures allégoriques et mythologiques découvrent au narrateur, un jeune étudiant en médecine, la machine du monde, depuis les règnes du minéral et du végétal jusqu'au corps humain. L'auteur sollicite les ressources discursives du mécanisme cartésien mais, conscient des limites de ce système explicatif à donner des images sensibles du vivant, il les rehausse d'images merveilleuses qu'il emprunte à l'imaginaire analogique et alchimique. Il est notable que les images les plus aptes, selon lui, à représenter le vivant relèvent du règne végétal. La plante serait alors une anti-machine.

The New Treatise on Physics, a little-known work by François-Joseph Hunauld (1701-1742), was published in 1742. Noteworthy for being his only attempt to adorn science with fictional elements, the New Treatise drew its main inspiration from Fontenelle's Conversations on the Plurality of Worlds and Pluche's Spectacle of Nature. In his account of a fantastic voyage with allegorical and mythical figures as guides, Hunauld intended to give readers a tour inside the great machinery of the world, from the mineral and the vegetable kingdoms to the human body. While sugarcoating his teachings with the narrative devices of the marvelous, Hunauld summoned the discursive resources of the expected references to Cartesian mechanical philosophy, which he quite unexpectedly intertwined with wondrous images borrowed from analogism and alchemy. Indeed, even though he remained a staunch defender of mechanicism, the physician perceived that machines failed to convey sensitive images of the living body. We argue that Hunauld found in traditional chemistry better tools for representing and understanding living matter, in the belief that chemical imagery and universal analogy reinsufflated life into a nature that mechanicians had robotized. Interestingly, the images that best fitted his conception of living matter pertained to the vegetable kingdom. 
INDEX

Mots-clés : voyage imaginaire, médecine, vitalisme, mécanisme, merveilleux, végétal, analogie, chimie, alchimie, Hunauld, Fontenelle, Pluche

Keywords : imaginary travel, medicine, vitalism, mechanism, marvel, vegetal, analogy, chemistry, alchemy, Hunauld, Fontenelle, Pluche

\section{AUTEUR}

SARAH BENHARRECH

University of Maryland 